\title{
DE LA BIOGRAFÍA A LA PROSOPOGRAFÍA: LOS PARLAMENTARIOS DE LA COMUNIDAD AUTÓNOMA VASCA (1977-1982)
}

\author{
ARITZ OnAINDia MARTÍNEZ \\ Vitoria-Gasteiz, 2018, Parlamento Vasco, 515 páginas \\ ISBN-978-84-939774-8-1
}

Nadie cuestiona hoy que el género biográfico está ya plenamente asentado y de moda en la historiografía contemporaneísta, en cierto modo, como reacción a la crisis de las "escuelas históricas" que había dominado la historiografía europea de la segunda mitad del siglo XX, como fueron la de los Annales, el materialismo histórico o la historia cuantitativa, tan seguida en el ámbito de la historia económica. Y al calor de esta recuperación de la biografía hay que situar también el auge que en estos momentos está teniendo la prosopografía, especialmente en los campos de la historia política o, más concretamente, en la historia parlamentaria.

Auténticos pioneros en esta tarea han sido los miembros del Grupo de Investigación de la Universidad de País Vasco "Biography \& Parliament" liderados por Joseba Agirreazkuenaga y Mikel Urquijo, en cuyo seno se han elaborado las metodologías más completas para afrontar este tipo de trabajos. Una buena prueba de lo que decimos es el libro recientemente publicado de Aritz Onaindia que vamos a comentar a continuación. Resultado de su tesis doctoral, este trabajo lo que hace es dar una continuidad cronológica a anteriores publicaciones que se han preparado en el seno del citado Grupo sobre los parlamentarios vascos, desde las Cortes de Cádiz a los tiempos de la Segunda República. Concretamente lo que aquí se realiza es la prosopografía de los parlamentarios vascos surgidos de las primeras elecciones de la reciente democracia -las de 1977- de las que le siguieron en 1979, de las primeras a la Cámara vasca en 1980 y de las nuevas generales del año 1982 que dieron la primera mayoría absoluta al Partido Socialista. Se analiza y se compara pues, la trayectoria de los 142 parlamentarios que representaron a las tres provincias vascas en este periodo, tanto de los electos, como de los que llegaron después como sustitutos al causar baja algunos de los titulares, así como de los senadores autonómicos elegidos por la Cámara vasca para que la representaran en el Senado. En definitiva, como nos dice nuestro autor, lo que el libro pretende es "radiografiar" la representación parlamentaria vasca de los primeros años de la democracia, tanto en las Cortes españolas como en el Parlamento de la Comunidad. 
No extraña pues, que el libro se abra con un completo estado de la cuestión sobre la trayectoria historiográfica de la prosopografía parlamentaria, en el que no sólo se comentan las principales aportaciones que en las últimas décadas se han realizado en la historiografía internacional, sino que también se da un repaso a lo que sobre este tema se ha venido publicando en los últimos años en las comunidades autónomas que hoy conforman el Estado español. A continuación, se explican las fuentes históricas que se han empleado, destacando especialmente el autor la importancia que para su investigación ha tenido la historia oral, dado que, como bien cuenta, ha podido entrevistar a casi todos los parlamentarios que se estudian, mientras que para los que ya han fallecido el acercamiento se ha realizado a través de personas "representativas" de sus ámbitos familiar y social. Por último, en este capítulo inicial se explica la metodología empleada, claramente deudora de los trabajos ya conocidos del Grupo citado de la UPV. Sin embargo, este libro va más allá del completo estudio prosopográfico que realiza, porque el capítulo cuarto del mismo -el que sigue al de las fuentes y metodología- es un detallado análisis del contexto histórico de la Transición Política y de sus principales hitos, como fueron la formación de las Cortes de 1977 y la aprobación de la Constitución al año siguiente, y también sobre la formación de las primeras asambleas autonómicas de España y el primer Parlamento vasco de la democracia. En este último aspecto, el autor destaca la singularidad que tuvo la Transición en el País Vasco, como el bajo apoyo que alcanzó el texto constitucional en el referéndum, la existencia de una violencia política en la que se mezclaron los atentados de ETA y el GAL y una fuerte conflictividad sociolaboral que arrancó con los sangrientos sucesos de Vitoria de 1976.

El capítulo quinto está centrado la descripción del marco político que servirá de base a la investigación prosopográfica que vendrá a continuación, al comentar el sistema electoral con el que se realizaron los comicios del periodo 1977-1982. En este sentido, el autor llama la atención sobre el contraste que hay entre las elecciones generales al Congreso de los Diputados y las que se celebraron para la Cámara vasca. Y es que si en las primeras se impuso el criterio proporcional de la población, de manera que si Bizkaia tenía diez diputados, a Gipuzcoa se le asignaron siete y a Araba cuatro, no ocurrió lo mismo en las del Parlamento vasco. Aquí, por las disposiciones estatutarias, el criterio demográfico no se tuvo en cuenta, fijándose en sesenta los diputados que lo formaban, a razón de veinte por cada provincia, a pesar de las diferencias de población que tenían entre sí. Se comentan a continuación los resultados obtenidos por las diferentes fuerzas políticas en estos cuatro procesos electorales, poniéndose de manifiesto la fortaleza del PNV, que resulta el partido más votado, marcando el claro contraste que se da entre las Cortes del Estado y el Parlamento vasco. Si en las 
primeras se da un bipartidismo imperfecto articulado sobre la UCD y el PSOE, en Euskadi emerge un mapa político sostenido sobre seis partidos y coaliciones, con dos importantes fuerzas nacionalistas -PNV y HB-, mientras que los dos partidos estatales -UCD y PSOEquedaban en una posición más debilitada, situándose en una posición todavía más subordinada, Euskadiko Ezquerra y Alianza Popular.

No obstante, es en el capítulo sexto -el más extenso- donde se vuelcan todos los datos que retratan a los 142 parlamentarios vascos de la Transición y que constituyen el objeto central de esta monografía. Las conclusiones que podemos extraer sobre los contornos sociológicos de esta élite política son verdaderamente esclarecedoras. En primer lugar, son políticos más jóvenes - con 42,5 años de media de edad- que los representantes de otras cámaras legislativas españolas y europeas con los que se comparan. Una juventud que pone en evidencia dos cosas: la primera, que al llegar la Transición se ha producido un claro relevo generacional en la $<<$ clase política $>>$ vasca y, en segundo lugar, que ésta ha afectado sobre todo a los principales fuerzas políticas de la Comunidad Autónoma como eran el PNV y el PSE-PSOE. Una situación que se veía todavía más acentuada en la Cámara vasca, donde el 66 por ciento de sus diputados no superaban los 40 años de edad (p. 235) y donde, además, se da una circunstancia que no tiene parangón en otros Parlamentos del Estado: que la ya citada violencia política va a provocar la muerte de hasta cinco de estos parlamentarios analizados, como fueron los casos de Enrique Casas (PSE-PSOE), Santiago Brouard (HB), Josu Muguruza (HB), Gregorio Ordóñez (AP) y Fernando Buesa (PSE-PSOE).

Otro rasgo destacado que muestra este estudio es el importante arraigo que tienen estos parlamentarios con los territorios por los que fueron elegidos, a pesar de que algunos no llegaron a nacer en esta Comunidad Autónoma porque llegarían de la emigración o del exilio. Una realidad que lleva a una característica que no se da en otros territorios: entre estos parlamentarios vascos del periodo 1977-1982 no hubo ningún "cunero" (pp. 256-257), a diferencia, por ejemplo, de lo que en estos años ocurría en Castilla-León. También destaca el estudio la importancia que tienen las redes familiares, hasta el extremo que 32 de ellos -el 25,3 por ciento- presentaban alguno o más familiares estrechamente ligados a algún tipo de cargo público. Destacan, por ejemplo, los hermanos Aguiriano y Múgica en el PSE-PSOE o la saga de los Orejas, que arrancó de los años de la República, continuó en las Cortes franquistas hasta llegar a los primeros parlamentos de la democracia.

En cuanto al género, hay que destacar que entre estos 142 parlamentarios sólo hubo nueve mujeres -el 6,3 por ciento- una cuota de participación femenina verdaderamente baja, pero que fue muy parecida a la que tuvieron otras asambleas autonómicas y nacionales de la 
época y que tiene mucho que ver con el tipo de sociedad que emergió en los primeros años de la Transición en la que la mujer apenas si tenía todavía presencia en el espacio público (p. 291).

Estos parlamentarios vascos, en su mayoría - un 80 por ciento- tenían estudios superiores y el Derecho era la licenciatura que más de repetía y también resulta interesante el apartado titulado "Renovación del personal parlamentario", porque pone de manifiesto que sólo tres de los 142 analizados tuvieron una actividad institucional en los años de la Segunda República. Eran los nacionalistas Jáuregui, Leizaola y Monzón, lo que evidenciaba la clara discontinuidad que hubo entre esta etapa democrática y la anterior, mientras que, por el contrario, algunos parlamentarios de los partidos de centro-derecha, como eran AP y UCD, sí presentaban vinculaciones con el periodo anterior de la dictadura franquista, eso sí, en un porcentaje más bajo -el 4,2 por ciento- que en las primeras Cortes democráticas de 1977, donde la presencia de estos procuradores del franquismo llegó hasta el 16,3 por ciento (p. $330)$.

Este último capítulo se cierra con un detallado análisis del protagonismo y la actividad que estos diputados y senadores vascos tuvieron tanto en las Cortes españolas como en su propio Parlamento y el libro se completa con un detallado apéndice que incluye la relación completa de estos parlamentarios, su distribución por partidos políticos y en los grupos parlamentarios de las tres cámaras legislativas, Congreso Senado y la Cámara vasca.

Aritz Onaindia ha elaborado un modélico trabajo de prosopografía parlamentaria, con un gran rigor metodológico y con un completo abanico de fuentes y el resultado de su investigación es un completo retrato de la élite política vasca que no sólo protagonizó la Transición en aquel territorio, sino que también contribuyó a crear institucionalmente su régimen autonómico, un libro que consideramos fundamental para conocer mejor la singularidad con la que se vivió en esta Comunidad Autónoma uno de los periodos históricos más interesantes del siglo XX español.

\section{Diego Caro Cancela}

Universidad de Cádiz 